\title{
FEDE E SACRAMENTI. L'INSEGNAMENTO DELLA TRADIZIONE
}

Ci sono tanti fenomeni negativi nella vita sacramentale che ci mostrano una relazione disturbata tra la fede vissuta e i sacramenti. Da una parte vediamo una crescente diminuzione della frequenza ai sacramenti nel mondo occidentale. D'altra parte la pratica sacramentale nel mondo occidentale spesso non è una consapevole espressione di fede. Vediamo tanti difetti dal punto di vista di fides qua ed anche da quello di fides quae. Il chiarimento della relazione fede/sacramenti è diventato una questione improrogabile. Noi dobbiamo trattare il tema nel contesto attuale della Chiesa, ma alla luce della tradizione della Chiesa. Prima di tutto è molto utile di analizzare le opere dei padri (cfr. I, II, III. capitoli). I Padri sono testimoni della fede dei primi secoli vitalmente inseriti nella tradizione che deriva dagli Apostoli. Essi infatti hanno congiunto sacra scrittura, teologia, liturgia, vita spirituale e pastorale in una unità vitale per cui le loro opere ci aiutano anche per la Chiesa del terzo millenio. La pristina Sanctorum Patrum norma (cfr. SC 50) è da ritenersi la norma e la regola ispiratrice anche per la Chiesa di oggi. Anche la sintesi scolatistica (Tommaso d'Aquino) ci aiuta per dare una risposta alla nostra domanda principale. Nella spiegazione di S. Tommaso vediamo come punto di partenza una tesi chiara: "La Chiesa è fondata sulla fede e sui sacramenti", e qualifica espressamente i sacramenti come sacramenta fidei. Dobbiamo conoscere anche la Riforma e l'insegnamento del concilio di Trento. Vedremo che l'analisi storica del rapporto tra fede e sacramenti ci aiuta la risposta a proposito del problema della Chiesa nei nostri giorni. Come conclusione finale vale la pena di analizzare il Sacrosanctum concilium n. 59.

Rev. prof. dott. hab. Lajos Dolhai - docente di teologia dogmatica e Rettore all'Istituto Superiore di Teologia di Eger (Ungheria), professore alla Facoltà Teologica dell'Università Cattolica Péter Pázmány di Budapest; membro della Comissione Teologica Internazionale di Roma; indirizzo di corrispondenza; e-mail: rektor@eghf.hu; ORCID: 0000-0002-2684-5218. 
Il rapporto tra la fede e i sacramenti è sempre stato al centro della riflessione teologica sin dall'epoca patristica (cfr. donatisti). ${ }^{1}$ Negli ultimi decenni il tema è ritornato nella sacramentaria. ${ }^{2}$ Il distacco dai sacramenti nei paesi occidentali è da tempo oggetto di riflessione della teologia contemporanea. ${ }^{3}$ Il chiarimento del tema è diventato una questione improrogabile, perché allo stesso tempo vediamo una crescente diminuzione della frequenza ai sacramenti e la mancanza della fede necessaria nella pratica sacramentale.

Dobbiamo vedere che l'approccio tradizionale del nostro tema è ormai superato. Purtroppo nella riflessione classica c'era spesso soltanto la dottrina delle condizioni minimali nell'attuazione di un sacramento. I concetti di validità, di fruttuosità del sacramento, con tutto il peso del loro senso giuridico e formale, indicano semplicemente che, nel riflettere sul rapporto fede e sacramenti, per lungo tempo «la Chiesa cattolica si è attestata così su un minimo che non rende ragione dell'evento sacramentale nel suo insieme». ${ }^{4}$

Noi dobbiamo trattare il tema nel contesto attuale della Chiesa, ma alla luce della tradizione della Chiesa. Prima di tutto molto utile di analizzare le opere dei padri. I Padri sono testimoni della fede dei primi secoli vitalmente inseriti nella tradizione che deriva dagli Apostoli. ${ }^{5}$ Essi attestano la vivificante presenza di questa Tradizione, le cui ricchezze sono trasfuse nella pratica e nella vita della Chiesa che crede e che prega. Essi infatti hanno congiunto sacra scrittura, teologia, liturgia, vita spirituale e pastorale in una unità vitale per cui le loro opere ci aiutano anche per la Chiesa del terzo millenio. La pristina Sanctorum Patrum norma ${ }^{6}$ è da ritenersi la norma e la regola ispiratrice anche per la Chiesa di oggi. Vedremo che l'analisi storica del rapporto tra fede e sacramenti ci aiuta la risposta a proposito del problema della Chiesa nei nostri giorni.

\section{LA VITA SACRAMENTALE DEI PRIMI SECOLI}

È chiaro che dall'inizio i sacramenti appartenevano alla vita della Chiesa ed all'identità cristiana. Nei padri si può scoprire la sacramentalità della fede cristiana,

L'opera più completa sulla storia del rapporto fede-sacramenti: L. Vilette, Foi et Sacrement, 2 voll., Paris 1964.

2 Cfr. C. Rochetta, I sacramenti della fede. Sacramentaria biblica fondamentale, vol. 1, Bologna 2001, 246-250; R. Gerardi, I sacramenti della fede cristiana, Roma 1985, 125-130; P.M. Gy, Problemes de theol. sacramentaire, La Maison Dieu 110 (1972), 124-142.

3 Cfr. L. Dolhai, Fede e sacramenti, Folia Athanasiana 17 (2015), 67-93.

4 H. Vorgrimler, Teologia dei sacramenti (rad. italiana), Queriniana (GDT 212), Brescia 1992, 116.

5 Cfr. L. Dolhai, Az ókeresztény egyház liturgiája, Budapest 2001, 9-21.

6 Cfr. SC 50; DV 8. 
la rilevanza dei sacramenti ed il rapporto speciale fra la fede e i sacramenti. Non per nulla nell'antico rito battesimale si dava grande importanza alla solenne professionale di fede. ${ }^{7}$ È nata già nella chiesa antica la regula fidei. Nel punto culminante della santa Messa il sacerdote dice: mysterium (= sacramentum) fidei. Non poche volte i Padri ricorrono a questa espressione per designare i sacramenti, e specialmente il battesimo ed eucaristia. ${ }^{8}$ I padri greci - specialmente Giovanni Crisostomo (†407) utilizzano spesso la concezione "mysterion" nell'ambito cultuale.

Il legame intrinseco tra la fede e il sacramento si manifesta anche nella terminologia. Tertulliano applicando la parola latina "sacramentum" al battesimo che egli considera come il giuramento per eccellenza, opposto agli obblighi dell'idolatria; ci sottolinea la rilevanza della decisione personale fondata nella fede. ${ }^{9}$ È ovvio per prendere una tale decisione, la persona ha anche bisogno di sapere qual è il contenuto della fede.

Di conseguenza, una catechesi completa precede il conferimento dei sacramenti ed è quasi parte costitutiva dell'integrazione nel corpo della Chiesa stessa, come dimostra lo sviluppo del catecumenato nella Chiesa antica. D'altra parte, la primitiva forma del battesimo era internamente collegata a una professione di fede in forma di dialogo - come attesta la Traditio Apostolica. ${ }^{10}$

In generale, la preparazione veniva presa molto sul serio. Il fatto che a volte alcuni documenti ecclesiastici si scagliassero contro la negligenza nella preparazione è una chiara testimonianza della preoccupazione per essa ${ }^{11}$. I candidati per il battesimo venivano esaminati riguardo alla sincerità della loro intenzione e del loro progresso (cf. gli "scrutini" della $2^{\circ}, 3^{\circ}$ e $4^{\circ}$ domenica prima di Pasqua). Naturalmente, si presumeva che la conversione e la fede del cuore avessero anche una parte "esterna" e visibile, consistente non soltanto nella professione del Symbolum, ma anche in uno stile di vita corrispondente al duplice comandamento dell'amore verso Dio e il prossimo, e nel collegamento vissuto con la Chiesa in preghiera.

Dall'inizio i cristiani erano convinti che il battesimo nel nome di Gesù aprisse l'ingresso alle comunità formate da coloro che invocano questo nome (cfr. ianua sacramentorum). In ogni caso che il battesimo è connesso con la fede in Cristo

7 De Baptismo, 6; Traditio Apostolica, 21 (conferimento del battesimo).

8 Cfr. C. Rochetta, Sacramentaria fondamentale, Dal "mysterion" al "sacramentum", Bologna 1989, 245-285.

9 Tertulliano, Ad mart., 3; De spect., 24,4; De idol., 19,2; De cor., 11,1.

10 Traditio apostolica, 15 (entrata nel catecumenato); 17-20 (processo del catecumenato); 21 (conferimento del battesimo).

11 Concilium Nicaen. Can. 2: Il Battesimo è stato conferito troppo presto, è necessaria una migliore preparazione; Pp. Siricius ep. Ad Himerium (385 d. C.), n. 3. 
e nella sua opera salvifica, perché il Signore dice: «chi crederà e sarà battezzato...» (Mc 16,16). Sulla orme di questo brano i Padri trattano la questione del rapporto fra fede e sacramento sopratutto quando trattano del battesimo. Tipico esempio lo troviamo nel testo De Baptismo di Tertulliano:

Ricordatevi che solo un fede completa e matura può essere sicura di ottenere la salvezza. La salvezza del battesimo si ottiene non imponendo soltanto il dito ma attraverso una fede sicura e matura. ${ }^{12}$

Lui risponde a qualcuno che mette in discussione il battesimo o messa in discussione la fede difendendo quest'ultima:

Ecco che quei miserabili, disgraziati sollevano obiezioni di questo tipo: il battesimo non è necessario, basta la fede. Abramo era un uomo gradito a Dio solo per la fede e non per il sacramento dell'acqua. Ebbene io rispondo, che in tutte le cose sono sempre le situazioni conclusive, quelle definitive e che quanto viene dopo ha più valore di quello che c'era prima. ${ }^{13}$

Anche a proposito dell'Eucaristia vediamo l'insegnamento chiaro dei padri. La Didaché si dimostra già esplicitamente:

Nessuno mangi, né beva della vostra eucaristia, se non i battezzati nel nome del Signore, poiché per questo il Signore ha detto: Non date le cose sante ai cani. ${ }^{14}$

Già nei primi secoli, per ricevere un sacramento (es. Eucaristia) è stato molto importante il contenuto della fede cristiana (fides quae). ${ }^{15}$ La fede deve corrispondere alla fede della Chiesa. ${ }^{16}$ Ireneo esprime la fede eucaristica nell'Adversus haereses: «Il nostro pensiero concorda perfettamente (symphonos) con l'eucaristia e l'eucaristia conferma a sua volta il nostro pensiero». ${ }^{17}$ Questa regola fidei si vede chiaramente nella prima Apologia di San Giustino:

Tertulliano, De Baptismo, 18,6.

Ibid., 13,1 .

Didaché, 9,5.

Cfr. Giustino si esprime in modo ancora più netto: «Questo alimento noi lo chiamiamo eucaristia, non è dato parteciparne se non a chi crede veri gli insegnamenti nostri, ha ricevuto il lavacro per la remissione dei peccati e la rigenerazione e vive secondo la norme di Cristo» (Apol. I, 66,1).

16 Cfr. Adv. Haer, I,10.

17 Ibid., IV, 18,5. 
A nessun altro è lecito partecipare all'Eucaristia, se non a colui che crede essere vere le cose che insegniamo, e che sia stato purificato da quel lavacro istituito per la remissione dei peccati e la rigenerazione, e poi viva cosi come Cristo ha insegnato. ${ }^{18}$

Nella Teofania anche Eusebio $(\dagger 340)$ indica le disposizioni necessarie per accostarsi all'eucaristia: «Con una coscienza illuminata, cun la purezza dell'anima e con la santità della vita». ${ }^{19}$ Efrem Siro $(† 373)$ nel quarto Sermone sulla Passio$n e$, così parafrasa le parole di Gesù: «Prendete, mangiate con fede, senza esitare, perché questo è il mio corpo e colui che lo mangia con fede mangia il fuoco dello Spirito che è in esso». ${ }^{20} \mathrm{Chi}$ «è degno si accosta, chi non è degno, si penta», è similmente nelle Costituzioni Apostoliche. ${ }^{21}$ Essa si riflette nell' invito liturgico "Il Santo ai Santi", commentato da Teodoro di Mopsuestia. Con i "santi" si intendono, come in san Paolo, in primo luogo i battezzati, naturalmente i battezzati che vivono con la Chiesa, come si può vedere soprattutto nei sermoni di Giovanni Crisostomo. ${ }^{22}$ Il "doctor eucharistiae" richiede ai suoi sacerdoti anche di respingere, se necessario, certe persone ${ }^{23}$ Allo stesso modo anche Agostino avverte chiaramente che il cibo sacramentale dona salvezza e vita soltanto se viene consumato "spiritualmente", con la fede circa il contenuto invisibile, e con una coscienza pura $^{24}$, cioè una vita che corrisponde all'amore di Cristo e dei suoi membri.

\section{LA CONTROVERSIA NEL III E IV SECOLO}

Le controversie sviluppatesi nel III e IV secolo, a proposito dei sacramenti dati o ricevuti dagli eretici, permisero di sottolineare come il valore e l'efficacia dei sacramenti dipendono dall'esistenza e dall'azione della Chiesa, chi li ha ricevuti da Cristo nella fede.

La sacramentaria di Sant'Agostino è una riflessione di fede su un momento particolare della vita della chiesa. Agostino, polemizzando con i donatisti, si trova quasi costretto a distinguere tra "sacramentum" (=segno esterno) e "res

Cap. 66-67 (PG 6, 427-431).

19 Theophaneia, $3,17$.

20 Sermones in hebdomadam sanctam, IV (Opera omnia, SChr 121, 137).

21 Costit. Apost., VII, 26: «Chi non è degno, dovrebbe essere ammesso nello stato dei penitenti, affinché possa diventare degno».

22 Hom. in Matth., 82, n. 4 (credere alla presenza reale); hom. 25, n. 3, hom. 7,6 Super Rom., hom. 9, n. 8 (carità)

23 In Matth. Hom., 82: responsabilità del sacerdote nel conferimento del sacramento.

24 Tract. in Ioh., 26, n. 11. 
sacramenti" (=dono interiore). I donatisti argomentavano: «Dove c'è battesimo, eucaristia e ordine, lì c'è la comunione con Cristo, lì si trova la chiesa; ma noi conserviamo infatti questi riti, perciò costituiamo la chiesa». Agostino risponde riconoscendo ai donatisti il "sacramentum", ma non la "res" ("aliud es virtus interna, aliud sacramentum") I donatisti hanno sì il sacramento, ma non la grazia di Cristo e quindi non sono la chiesa vivente di Dio.

Negli scritti antidoceti sottolinea come il battesimo possa essere celebrato senza che si ricevano i doni salvifici da esso significati; «una cosa infatti è possedere il sacramento del battesimo e un' altra è riceverne l'effetto». ${ }^{25}$ È questa distinzione la chiave di fondo della dottrina sacramentale agostiniana: essa ritorna nella teologia, al di fuori di dispute particolari, ${ }^{26}$ e riappare implicitamente nell' analisi della confermazione e dell'ordine. ${ }^{27}$

Anche a proposito dell'Eucaristia vediamo questa distinzione chiara. C'è una differenza fra il piano significante e il quello significato. Dunque, anche sul livello dei singoli sacramenti vediamo la inseparabilità ed allo stesso modo la non-identità delle due dimensioni. ${ }^{28}$ Riprendendo il linguaggio dei grandi scolastici, possiamo dire che se la res significata e contenuta dai segni sacramentali è il dono dello Spirito e la sua grazia, la res significata, ma non contenuta è la carità di Dio-Trinità che edifica l'unità della Chiesa.

Nella sua lotta contro i donatisti Agostino doveva affrontare una posizione intransigente. Non esita per niente a riconoscere il principio della necessità del battesimo ${ }^{29}$. Insiste pure nello stesso scritto anti-donatista sulla tradizione della Chiesa universale secondo la quale i bambini che non sono ancora incapaci di confessare la loro fede non vengono inutilmente battezzati: anche loro ottengono per mezzo del battesimo la salvezza. ${ }^{30}$ Agostino ricorda dunque continuamente che noi non dobbiamo affidarci agli uomini, ma dobbiamo mettere tutta la nostra speranza nel Signore. E Cristo che battezza, non cessa di ripetere, non Pietro, non Paolo, non Giuda. ${ }^{31}$

25 Cfr. De bapt. Contra Don., 5,21,29.

26 Cfr. ad esempio Tract. in Joh. 26,11 dove si impiega la terminologia sacramentum/virtus sacramenti: «Nam et nos hodie accipimus visibilem cibum, sed aliud est sacramentum, aliud virtus sacramenti».

27 Cfr. ad esempio: In Ep. Joan. Tr., 4,10 per la confermazione; De baptismo. I, 1,2 per l'ordine. Anche noi oggi riceviamo un cibo visibile: ma altro è il sacramento, altra è la virtù del sacramento. Quanti si accostano all'altare e muoiono, e, quel che è peggio, muoiono proprio perché ricevono il sacramento! È di questi che parla l'Apostolo quando dice: Mangiano e bevono la loro condanna $(1$ Cor 11, 29). Non si può dire che fosse veleno il boccone che Giuda ricevette dal Signore. E tuttavia non appena lo ebbe preso, il nemico entrò in lui; non perché avesse ricevuto una cosa cattiva, ma perché, malvagio com'era, ricevette indegnamente una cosa buona» (Tract. In Joh., 26,11).

29 Bapt., II, 14, 19.

30 Cfr. ibid., IV, 23-30.

31 Ved. Tract. in Ioh., 5,11ss. 
Per riconciliare i dati tradizionali concernenti la necessità assoluta del battesimo e la sua visione della Chiesa universale, Agostino introduce in particolare la distinzione fra la communio sacramentorum e la societas sanctorum. Ogni uomo deve avere la possibilità di essere battezzato, senza dipendere dallo stato morale del ministro del battesimo. Perciò il Signore stesso si fa garante della santità del battesimo. Questo dunque è sempre valido, se viene celebrato secondo la forma del Signore. ${ }^{32}$ Per mezzo della consacrazione battesimale il credente appartiene alla communio sacramentorum, ma solo credendo pienamente in Cristo, Signore di tutti i credenti, fa anche parte della societas sanctorum.

\section{LA TEOLOGIA DEI PADRI GRECI}

La teologia dei padri greci, mette in luce molto vigorosamente il ruolo insostituibile della fede personale per la partecipazione fruttuosa al "mistero" dell'azione del Cristo nel sacramento. La fede che si esprime in una autentica conversione è la necessaria via di accesso a questo mistero; nell'azione sacramentale questa fede è nutrita e consacrata; e il credente è marchiato dal "sigillo della fede". Un testo significativo per il nostro tema si trova anche in S. Basilio.

La fede e il battesimo sono i due modi della salvezza, l'uno all'altro congiunto e inseparabili. La fede infatti si perfeziona col battesimo, il battesimo si fonda sulla fede e l'una e l'altro raggiungono il compimento perfetto mediante gli stessi nomi. Come infatti crediamo nel Padre e Figlio e Spirito Santo, così anche battezziamo nel nome del Padre e del Figlio e dello Spirito Santo. Viene prima la professione che porta alla salvezza, segue subito appresso il battesimo, a suggellare il nostro assenso..$^{33}$

La catechesi sacramentaria o mistagogica ci ammonisce che dobbiamo recuperare l'unità interna tra il creduto, il celebrato e il vissuto sacramentale, superando ogni forma di dicotomia o dualismo tra rito e vita. ${ }^{34}$ Cirillo di Gerusalemme sostiene fortemente l'importanza del cambiamento del cuore e avverte: «Se la vostra intenzione rimane sbagliata [...], allora, infatti, l'acqua ti accoglie, ma non lo Spirito Santo» ${ }^{35}$. Ciò che viene richiesto esplicitamente è non tanto la forza della fede, nel senso di un carisma (una fede tangibile e intensa che può spostare le montagne), quanto il consenso fedele all'annuncio fatto della

32 Cfr. Bapt., II, 10,15; IV 12,18.

33 Basilio, De Spiritu Sancto, XII, 28.

34 Cfr. Ambrogio, De mysteriis, 1 e 2.

35 Procatechesis, Introduzione n. 4 (PG 33,340A). 
Chiesa: «Hai bisogno della fede, questo dipende da te, della fede in Dio, affinché tu ottenga la fede che Dio dà e che opera cose sovrumane». ${ }^{36}$ La fede può e deve crescere; la predisposizione a questo è parte integrante della decisione per il battesimo. ${ }^{37}$

La catechesi sui sacramenti cominciava in effetti dopo che essi erano stati conferiti ai nuovi iniziati. Vi sono in questo due ragioni essenziali. Si pensava anzitutto che i "misteri" della fede, a motivo della legge dell'arcano, non dovessero essere svelati interamente se non ai cristiani: i sacramenti esprimono la piena realizzazione di questi misteri di fede nella vita della Chiesa. Si pensava, d'altra parte, che per comprenderli bene, valesse meglio prima viverli e poi riceverne la spiegazione ${ }^{38}$ Dunque, accostarsi ai sacramenti d'iniziazione non dovevano avere un fede matura con il tutto il contenuto della fede cristiana. È stato più importante il "fides qua" che il "fides quae". La fede può e deve crescere; la predisposizione a questo è parte integrante della decisione per il battesimo.

Anche la liturgia come "lex credendi" sottolinea la rilevanza della fede nella vita sacramentale. Nelle tradizioni liturgiche, specialmente in Oriente, si può chiaramente riconoscere questo rapporto tra la fede, l'amore e la ricezione dell'Eucaristia, come nell'acclamazione prima della comunione del popolo: «Con timore di Dio, fede e amore accostatevi!» Nella liturgia di san Giovanni Crisostomo il diacono, il sacerdote e tutti i fedeli esprimono prima della Comunione una confessione esplicita verso Cristo che è presente con il suo corpo e il suo sangue: «Io credo, Signore, e confesso che tu sei in verità il Cristo, il Figlio del Dio vivente. Io credo anche che questo sia il tuo stesso corpo senza macchia e il tuo stesso sangue prezioso». ${ }^{39}$ Secondo la liturgia latina il fedele deve pregare con umiltà e fede la supplica del centurione: «Domine non sum dignus ut intres sub tectum meum: sed tantum dic verbo, et sanabitur anima mea». ${ }^{40}$

\section{LA SINTESI DELLA SCOLASTICA}

La sistematizzazione avviata fin dal $\mathrm{V}$ secolo è ripresa in una sintesi dottrinale ampia e profonda nel periodo scolastico.

$36 \quad$ Ibid., V, 10s.

37 Procatech., I,6; I,4 (essere fruttifico). Il monito contro la negligenza e la tiepidezza si trova anche nelle Catechesi di Giovanni Crisostomo ai Neo-Battezzati: Cath. 7,7; 9,2.7.21.26; 10,1.2; 12,24 etc.

38 Cfr. Cirillo di Gerusalemme, Catechesi, 19,1.

39 La divina liturgia di Giovanni Cristostomo, Comunione dei fedeli.

40 Messale Romano, Riti di comunione. 
Infatti, se chiediamo alla parte generale del trattato sui sacramenti della Summa Theologica di presentarci il rapporto tra fede e sacramenti restiamo in prima istanza abbastanza delusi: il tutto il trattato, infatti, il tema che cerchiamo non c'è. E piuttosto occasionalmente leggiamo qualcosa di esplicito sulla differenza e la complementarità tra fede e sacramento, oppure dobbiamo cercare altrove ciò che è importante per il nostro tema. ${ }^{41}$

S. Tommaso qualifica espressamente i sacramenti come "sacramenta fidei" e "signa protestantia fidem". ${ }^{42}$ Nella sua spiegazione vediamo come punto di partenza una tesi chiara: "La Chiesa è fondata sulla fede e sui sacramenti». ${ }^{43}$

Egli chiama il battesimo "sacramento della fede", e spiega il rapporto fra la passione di Cristo, la fede e il battesimo. ${ }^{44}$ Secondo la ben fondata tesi dell'Aquinate la fede trova la massima espressione della sua efficacia nell'incontro sacramentale. ${ }^{45}$ Per lui i sacramenti, affidati da Cristo alla Chiesa, sono i segni rituali della fede cristiana, e hanno lo scopo di rappresentare e rendere attuali per il cristiano i misteri della redenzione, ai quali la Chiesa aderisce per mezzo della fede. La Chiesa indende il conferimento dei sacramenti a coloro che credono. Senza la fede "non eset remissio peccatorum" ${ }^{46}$ Pertanto, la Chiesa pone anche delle domande riquardo alla fede del ricevente. ${ }^{47}$

A prima battuta è facile scoprire come ciò che noi cerchiamo, nella Summa possiamo trovarlo anzitutto nella questio 61 , della parte III, dedicata al tema $D e$ necessitate sacramentorum. ${ }^{48}$ Sacramento e fede -intendendo sopratutto una fede animata dalla speranza e dalla carità - formano un tutto indissolubile nell'ordine concreto da S. Tommaso:

Riceviamo la salvezza mediante la fede in Cristo, che è nato ed ha patito per noi. Ma i Sacramenti sono segni che annunciano e manifestano quella fede per la quale l'uomo viene giustificato. ${ }^{49}$

413 testi significativi: S.Th., III, q. 61, a. 4 (i sacramenti come quaedam fide protestationes); S.Th., III/a, q. 62, a. 6. corpus (virtus passsionis Christi copulatur nobis per fidem et sacramenta). È molto simile il contenuto in III/a, q. 49, a. 3, ad 1; In Ep. ad Hebraeos, 3, lect. 3 (partecipiamo alla grazia mediante la fede e sacramenti della fede).

42 S.Th., III, q. 61. a. 4; cfr. ancora S.Th., III, q. 72, 5 ad 11.

43 In 4 Sent., dist. 17, q. 3, a. 1.

44 S.Th., III, q. 62, a. 5.

45 In 4 Sent., dist. 4, q. 1, a. 2.

46 S.Th., III, q. 62, a.5 ad 2.

47 S.Th., III, q. 68, a. 8 ad 2.

48 S.Th., III, q. 68, a. 1.

49 S.Th., III, q. 61, a. 4. 
Ma anche altrove, incontriamo un passo esplicitamente dedicato al tema che ci riguarda da vicino. Nel passo che ci interessa Tommaso afferma:

Virtus passionis Christi copulatur nobis per fidem et sacramenta, differenter tamen: nam continuatio quae eset per fidem, fit per actum animae, continuatio autem quae esset per sacramenta, fit per usum exteriorum rerum. ${ }^{50}$

Come si vede, l'uomo entra in contatto (copulatur) con il Redentore "per fidem et sacramenta". Tutte e due sono importanti. La fede è un atto spirituale-mentale, ma quella ha bisogno di un segno visibile come un segno della fede.

\section{LA RIFORMA E IL CONCILIO DI TRENTO}

Il periodo importante, che parte dalla Riforma, registra un totale rovesciamento di prospettive. I Riformatori vedevano nei sacramenti soltanto un'espressione della fede per raggiungere le promesse divine e insegnavano che erano stati istituiti soltanto per nutrire e confermare la fede. Tutta la loro efficacia dipende dal fatto che vengono ricevuti con fede (opus operantis).

Lutero unilateralmente esaltò la potenza giustificatrice della fede ("sola fides"intesa spesso nel senso individuale di fede-confidenza), a danno dell'efficacia propria dei sacramenti. ${ }^{51}$ È nella sola fede, "in occasione del sacramento", ma indipendentemente da esso (non sacramentum, sed fides sacramenti iustificat), che la giustificazione a la grazia divina raggiungono direttamente il credente, il quale non offre d'altronde nessun concorso.

L'insegnamento di Lutero esprime una dissociazione e una opposizione fra la fede e sacramenti. La teologia cattolica, opponendosi ai Riformatori, per lungo tempo, nella sua reazione, ha finito forse con l'esaltare unilateralmente il valore dei sacramenti, attenuando il ruolo della fede salvifica. Anche la teologia cattolica, in reazione ai riformatori, è caduta di tipo metodologico: ha talmente accentuato i sacramenti da trascurare il ruolo della fede. La sacramentaria post-tridentina ha quasi dimenticato il collegamento (con la fede); concentrandosi sul sacramento, facendogli assumere una posizione solitaria nell'universo teologico, dimenticando il collegamento con la fede, oppure limitando la fede.

$50 \quad$ S.Th., III, q. 62, a. 6. corpus.

51 Cfr. R. Gerardi, I sacramenti della fede cristiana, Roma 1985, 127-128. 
«Tridentinum Tridentino interpretandum est!» L'insegnamento del Tridentino dobbiamo vedere nel contesto della Riforma. ${ }^{52}$ Il concilio preoccupato di salvare i sacramenti. Il tema del rapporto fede-sacramenti non è trascurato dal concilio, ma è trattato con l'intento prevalente di affermare l'indispensabilità dei sacramenti. Infatti, sebbene nella sessione VI si affermi che la fede è "fundamentum et radix iustificationis" (DH 1532), nella sessione VII il tema del rapporto fede-sacramenti è visto solo come problema dell'insufficienza salvifica della fede senza il completamento dei sacramenti (DH 1600-1605), e in particolare, come il problema della necessità della fede per l'efficacia dei sacramenti: cioè il ruolo dell'opus operantis rispetto all'opus operatum.

Definendo l'efficacia dei sacramenti ex opere operato, il Tridentino (DH 1608) non intese certamente negare la necessità della fede per essere giustificato $(\mathrm{DH}$ 1529) e per ricevere il sacramento; né intese negare l'efficacia psicologia dei sacramenti. La teologia cattolica è però fedele nell'affermare, da una parte l'efficacia propria dei sacramenti istituiti da Cristo, e, dall'altra, la necessità assoluta della fede personale perché l'efficacia sia tale per il soggetto. Il sacramento è un atto di Cristo, un segno rituale efficace della grazia che viene da Cristo; e la definizione tridentina dell'efficacia oggettiva, in realtà non fa altro che dire questa verità propria della tradizione ecclesiale. Il sacramento è efficace (ex opere operato) non dipende dalla fede soggettiva degli individui personali (ex opere operantis).

È ovvio che nella sacramentaria attuale dobbiamo sottolineare meglio la forte interdipendenza che deve esserci fede e sacramenti, e questo deve avvenire col massimo equilibrio perché se ci spostiamo in maniera unilaterale sulla fede, il rischio è quello di cadere in una sorta di pelagianesimo, se ci spostiamo eccessivamente sul sacramento trascurando la fede, il rischio è quello di cadere nel "magismo" sacramentale.

\section{LA DOTTRINA DEL SACROSANCTUM CONCILIUM}

Il concilio Vaticano II - dopo la definizione dei sacramenti - ha messo in evidenza chiaramente le relazioni che passa fra la fede e i sacramenti: «Non sono lo suppongono la fede, ma, con le parole e gli elementi rituali, la nutrono, la irrobustiscono e la esprimono: perciò, vengono chiamati sacramenti di fede» (SC 59). ${ }^{53}$

52 Cfr. L. Krause, Der Sakramentenbegriff des Konzils von Trient und die heutige Sakramententheologie, Theologie und Glaube 45 (1955), 401-422.

53 Cfr. H. Schmidt, La costituzione sulla sacra liturgia, Roma 1966, 274-293. 
Questo testo riprende l'idea di S. Tommaso ${ }^{54}$ per dire che la fede non copre solo un momento del processo sacramentale, ma attraversa tutto il processo sacramentale e cioè prima, durante e dopo ${ }^{55}$ Nella stessa celebrazione sacramentale, la fede cresce, si rafforza e si esprime. La fede deve entrare nel processo del sacramento. Nel testo conciliare vediamo tre punti salienti:

I sacramenti suppongono la fede in un duplice senso: come "accesso" al mistero sacramentale (se manca la fede il sacramento appare solo come un simbolo esteriore o un rito vuoto, col rischio di diventare presto solo un gesto magico); come condizione necessaria (conditio sine qua non) perché il sacramento operi soggettivamente i doni che oggettivamente contiene.

I sacramenti manifestano la fede del soggetto e della Chiesa. La celebrazione dei sacramenti è una professione di fede vissuta. I Sacramenti sono segni con cui si professa la fede dalla quale l'uomo viene giustificato. Già il battesimo costituisce un'adesione di fede e l'inizio di un'esistenza nuova nella grazia della fede. La parola sacramentale vuole la risposta della fede del credente che, per via di essa, apprende e riconosce il mistero che essa opera.

I sacramenti alimentano la fede a due livelli fondamentali: in quanto comunicano il dono della grazia divina che realizza o rafforza la la vita cristiana del credente, ed in quanto sono celebrazioni che, mentre operano significano il mistero della salvezza, educando alla fede e alimentandola di continuo, come dice il testo conciliare: «Con le parole e gli elementi rituali la nutrono e la irrobustiscono».

\section{CONCLUSIONE}

Dopo aver fatto l'analisi, dobbiamo costatare che alla fede cattolica (cristiana) appartengono i sacramenti, ma non possiamo dimenticare mai, che i sacramenti suppongono la fede $!^{56}$ «Se è vero che i Sacramenti sono i Sacramenti della fede, (SC 59) si deve anche dire che la fede ha una struttura sacramentale». ${ }^{57}$

Fra fede e sacramenti corre una stretta relazione. Si tratta di un'interdipendenza, cioè riconoscere che Dio non agisce ad di fuori della libertà umana. ${ }^{58}$ Nessuno dei

54 Cfr. S.Th., III, q. 49, 3 ad 1; 62, 6; 72, 5 ad 1.

55 Cfr. E.J. Lengeling, Die Konstitution des zweiten Vatikanischen Konzils über die heilige Liturgie, Münster 1964, 134-135.

56 Cfr. K.H. Menke, Sakramentalität. Wesen und Wunde des Katholizismus, Regensburg 2012, 7-11; K. Góźdź, Teologiczne rozumienie matżeństwa osób ochrzczonych a niewierzacych, RT LXVI,2 (2019), s. 27.

57 Francesco, Lumen fidei, 40.

58 Cfr. Lexikon der kath. Dogmatik, ed. W. Beinert, Freiburg im Breisgau 1977, 447. 
grandi teologi ha messo mai in discussione questo dato sottolineato in rapporto fra la libertà dell'uomo data dalla risposta libera della Chiesa e il sacramento, cioè la grazia proveniente di Dio.

Anche la teologia cattolica, in reazione ai riformatori, è di tipo metodologico: ha talmente accentuato i sacramenti da trascurare il ruolo della fede. La sacramentaria post-tridentina ha quasi dimenticato il collegamento (con la fede); concentrandosi sul sacramento, facendogli assumere una posizione solitaria nell'universo teologico, dimenticando il collegamento con la fede, oppure limitando la fede.

Nella sacramentaria attuale dobbiamo sottolineare meglio la forte interdipendenza che deve esserci fede e sacramenti, e questo deve avvenire col massimo equilibrio perché se ci spostiamo in maniera unilaterale sulla fede, il rischio è quello di cadere in una sorta di pelagianesimo, se ci spostiamo eccessivamente sul sacramento trascurando la fede, il rischio è quello di cadere nel "magismo" sacramentale.

Un altro problema: di quale fede si sta parlando? È una fede personale? Una fede della Chiesa? Una fede iniziale, forte, matura? È ovvio che si tratta della fede della Chiesa. Il principio che guida sempre i Padri sulla sacramentaria è che essi sono celebrati nella fede della Chiesa, sono affidati alla Chiesa. Non possiamo dimenticare che il credere è un atto ecclesiale. Possiamo parlare della "forma ecclesiale della fede" ${ }^{59}$ La fede della Chiesa precede, genera, sostiene e nutra la nostra fede. ${ }^{60}$

Dunque, solo chi appartiene alla Chiesa e celebra nella fede della Chiesa può celebrare in verità e ricevere i frutti della salvezza. Ma poiché ogni credente è personalmente implicato nella definizione della Chiesa, allora necessità anche la fede personale. Dunque, gli adulti non possono accedere al sacramento senza la fede, senza l'adesione a quella parola che introduce al sacramento e ne svela il significato.

\section{BIBLIOGRAFIA}

Chauvet L.-M., Linguaggio e simbolo. Saggio sui sacramenti, Torino 1988.

Courth F., Die Sakramente. Ein Lehrbuch dür Studium und Praxis der Theologie, Breisgau 1995. Il cristianesimo delle origini. I padri apostolici, ed. A. Lenzubi, Bologna 2001.

Daniélou J., La catechesi nei primi secoli, Torino 1982.

Didier R., Les sacraments de la foi, Paris 1975.

La divina liturgia del santo nostro padre Giovanni Cristostomo, Roma 1967.

Dolnai L., Lex orandi, lex credendi, Teológia 34,3-4 (1999), 97-107.

59 Francesco, Lumen fidei, 22.

60 Cfr. CCC, 181. 
Dolnai L., Az ókeresztény egyház liturgiája, Budapest 2001.

Dolnai L., A szentségek teológiája, Budapest 2011.

Dolnai L., Fede e sacramenti, Folia Athanasiana 17 (2015), 67-93.

Gánóczy S., Bevezetés a katolikus szentségtanba, Pannonhalma 2006.

Gerardi R., I sacramenti della fede cristiana, Roma 1985.

Góźdź K., Teologiczne rozumienie matżeństwa osób ochrzczonych a niewierzacych, RT LXVI,2 (2019), s. 19-34.

Grillo A., Fede e sacramenti: questione classica e riformulazione contemporanea, in: Corso di Teologia sacramentaria, ed. A. Grillo, M. Perroni, P. Tragan, Roma 2000.

Gy P.M., Problemes de theol. sacramentaire, La Maison Dieu 110 (1972), 124-142.

Handbuch der Dogmatik, ed. Th. Schneider, vol. 2, Düsseldorf 1992.

Incontrare Cristo nei sacramenti, ed. L. Hubert, Roma 1988.

Krause L., Der Sakramentenbegriff des Konzils von Trient und die heutige Sakramententheologie, Theologie und Glaube 45 (1955), 401-422.

Lengeling E.J., Die Konstitution des zweiten Vatikanischen Konzils über die heilige Liturgie, Münster 1964.

Lexikon der kath. Dogmatik, ed. W. Beinert, Freiburg im Breisgau 1977.

Lies L., Sakramententheologie: eine personale Sicht, Wien 1990.

Menke K.H., Sakramentalität. Wesen und Wunde des Katholizismus, Regensburg 2012.

Le preghiere dei padri, ed. G. Bebnabei, Bologna 1979.

Rochetta C., Sacramentaria fondamentale, Bologna 1989.

Rochetta C., I sacramenti della fede. Sacramentaria biblica fondamentale, vol. 1, Bologna 2001, 246-250.

Schmidt H., La costituzione sulla sacra liturgia, Roma 1966.

Testa B., Die Sakramente der Kirche, Paderborn 1997.

Vilette L., Foi et Sacrement, 2 voll., Paris 1959/1966.

Vorgrimler H., Teologia dei sacramenti, Brescia 1992.

Parole chiave: distacco fra fede e sacramenti, sacramentalità, sacramentum fidei, fides quae, fides qua, sintesi scolastica, opus operantis - opus operatum, Sacrosanctum concilium n. 59

\section{FAITH AND SACRAMENTS. THE TEACHING OF TRADITION}

\section{Summary}

There are many negative phenomena in the sacramental life which point out to a disturbed relationship between lived faith and the sacraments. On one hand, we see a growing 
decrease of attendance to the sacraments in the western world. On the other hand, the sacramental practice in the western world is often not a conscious expression of faith. We see many flaws from the point of view of the fides qua and from that of the fides quae as well. The explanation of the faith-sacraments relationship has become an urgent question. We should deal with the topic in the actual context of the Church, but in the light of the Church's tradition. First of all it is very useful to analyze the works of the Fathers (cfr. chapters I, II, III). The Fathers are witnesses of the faith of the first centuries vitally inserted in the tradition which derives from the Apostles. In fact they have joined together sacred scripture, theology, liturgy, spiritual and pastoral life in a vital unity through which their works can help us and also the Church of the third millenium. The pristina Sanctorum Patrum norma (cfr. SC 50) is to be considered the norm and the inspiring rule for the Church of today as well. Also the scholastic synthesis (Thomas Aquinas) helps us to give an answer to our main question. In the explanation of St. Thomas we find a clear thesis as a point of departure: "The Church is founded upon faith and its sacraments", and expressly qualifies the sacraments as sacramenta fidei. We should also know the Reformation and the teaching of the Council of Trent. We will see that the historic analysis of the relationship between faith and the sacraments helps us to find the answer to the subject of the Church's problem in our days. As a final conclusion it is worth analyzing the Sacrosanctum concilium n. 59.

Keywords: separation between faith and sacraments, sacramentality, sacramentum fidei, fides quae, fides qua, scholastic synthesis, opus operantis - opus operatum, Sacrosanctum concilium, n. 59

\section{WIARA I SAKRAMENTY W NAUCZANIU TRADYCJI}

\section{Streszczenie}

W obecnym czasie pojawia się wiele negatywnych zjawisk w przestrzeni życia sakramentalnego, które świadczą o zaburzonej relacji pomiędzy życiem wiary a sakramentami. $\mathrm{Z}$ jednej strony w świecie zachodnim widzimy spadek liczby wiernych przystępujących do sakramentów. Z drugiej zaś strony sama praktyka sakramentalna w świecie zachodnim często nie jest świadomym wyrażaniem wiary. Widać dziś w związku z tym wiele niedostatków zarówno na płaszczyźnie fides qua, jak i na poziomie fides quae. Dlatego właśnie wyjaśnienie relacji pomiędzy wiarą a sakramentami staje się obecnie zadaniem szczególnie pilnym. Z tematem tym powinniśmy się zmierzyć w kontekście współczesnego Kościoła, ale w świetle jego Tradycji. Przede wszystkim pożyteczne jest przeanalizowanie pod tym kątem dzieł ojców Kościoła (por. części I, II i III). Ojcowie są świadkami wiary z pierwszych wieków, ulokowanymi w nurcie żywej Tradycji, sięgającej apostołów. 
W swoim nauczaniu łączyli oni przesłanie Biblii, teologię, liturgię oraz życie duchowe i duszpasterstwo w ramach jednej syntezy, poprzez którą ich teksty mogą służyć także nam i Kościołowi trzeciego tysiąclecia. Pristina Sanctorum Patrum norma (KL 50) powinna zostać przyjęta także dziś jako zasada inspirująca Kościół. Również synteza scholastyczna (Tomasz z Akwinu) pomaga nam odpowiedzieć na wywołane pytanie. W wyjaśnieniu Tomasza znajdujemy klarowną tezę, będącą punktem wyjścia: „Kościół jest zbudowany na wierze i jej sakramentach" - gdzie wprost mowa jest o sakramentach jako sacramenta fidei. Trzeba także odwołać się do reformacji oraz nauczania Soboru Trydenckiego. Zobaczymy wówczas, że historyczna analiza relacji pomiędzy wiarą a sakramentami pomoże nam odpowiedzieć na pytania nurtujące Kościół w naszych czasach. W konkluzji poddany analizie został 59 punkt konstytucji Sacrosanctum concilium.

Słowa kluczowe: odseparowanie sakramentów od wiary, sakramentalność, sacramentum fidei, fides quae, fides qua, synteza scholastyczna, opus operantis - opus operatum, Sacrosanctum concilium n. 59 